\title{
HEART WORM IN A LIONESS
}

\author{
S. Haque and Ansar Ahmad \\ Department of Veterinary Medicine, Ranchi Veterinary College, Kanke, \\ Ranchi 834006, India.
}

Dirofilaria immitis is encountered in canids and felids in tropical and subtropical regions. The parasite is transmitted as microfilaria by mosquitoes. The microfilaria develops in the host tissues before migrating into the venous system. The immature worms grow over a period of two to three months, causing arteritis of the pulmonary vessels which may cause fibrosis and pulmonary hypertension. The resulting clinical signs are coughing, dyspnoea, and exercise intolerance, right-sided cardiac enlargement and failure may ensure. It is fatal always in the case of heavy infestation but many infested dogs have shown few or no signs of illness in mild infestation. The most reliable diagnostic test is the detection of larvae (Microfilaria) in stained blood smear.

One lioness of Birsa Biological Park, Ranchi showed symptoms of weak hind quarters, occasional convulsive seizures, dyspnoea, complete loss of appetite, exercise intolerance, gradual weight loss, harsh dry coat, dehydration and anaemia. The lioness was moved into a squeeze cage and thoroughly examined. Body temperature was recorded morning and evening abd it was found to vary between $100^{\circ}$ to $101.5^{\circ} \mathrm{F}$. Heart and lungs were ausculted. Heart beat had increased, lungs could not be heard clearly due to groaning of the animal. Mucous membranes were pale, peripheral blood was examined for the presence of any parasite and was found negative for microfilaria. On analysis of blood collected from saphenous vein for blood urea nitrogen (BUN) and SGPT it was found that SGPT was 38IU. BUN was very high $(126.5 \mathrm{mg} / \mathrm{dl})$ and was approximately three times higher than the normal value (10-40mg/dl). On DLC examination neutrophil was found extremely high and it was $93 \%$. Urine culture was found positive for many colonies of non-fermenting bacilli and drug sensitivity test showed that the organism was sensitive to Amikacin (+++). Age and body weight was recorded which was approximately 17 years and 1.5 quintals respectively. The animal was very closely observed for symptoms. It was found that roaring was completely absent even at the time of roaring of others lions which is considered a most important symptom of ailment. The response to attendants' signals was very poor.

Fluid therapy, suitable antibiotic at recommended dose and

supportive therapy were given. On the fourth day of treatment the lioness started taking food but only chicken (around $2 \mathrm{~kg}$ per day). Gradually the diet increased to beef also. During the course of treatment convulsive seizure was noticed twice. The animal could not bear her body weight on her legs despite several efforts to make her stand. The BUN level returned to almost normal level after continuing treatment for seven days but the neutrophils (\%) level decreased only up to 82 .

Gradually the lioness became laterally recumbent, stopped taking food and collapsed after 35 days of onset of first symptoms. On postmortem examination it was found that her heart was full of adult heart worms, especially the pulmonary artery which was completely packed with Dirofilaria immitis. Liver and kidney were highly inflammed with necrotic foci which is in accordance with the finding of Baily (1963). Since the animal was weak, debilitated and dehydrated chances of secondary bacterial infection was high thereby increasing the neutrophil count. The BUN might have increased due to severe stress on the kidney resulting from severe dehydration (Chakrabarti, 1994).

The reason for not detecting microfilaria in the blood smear may be due to the occult state of heart worms. The animal may have many adult heart worms but no circulating microfilaria. Another reason may be because in some cases a negative result of blood smear can not be relied upon to identify infestation.

\section{Acknowledgement}

The authors are thankful to the District Forest Officer, Birsa Biological Park, Ranchi for inviting me to examine the ailing lioness and also for allowing to collect blood and urine samples for investigation.

\section{References}

Baily, W.S. (1963). Parasites and cancers: sarcoma in dogs associated with Spirocerca lupi. Arm N.Y. Academy 108: 890-932.

Chakrabarti, A. (1999). Text Book of Clinical Medicine. II revised edition. Kalyani Publishers, New Delhi, 124pp. 